\title{
$A{ }^{12} \mathrm{CO}(\mathrm{J}=1 \rightarrow 0)$ study towards the Ara OB1 region
}

\author{
E. M. Arnal ${ }^{1,2, \star}$, J. May $^{3}$, and G. A. Romero ${ }^{1, \star \star}$ \\ 1 Instituto Argentino de Radioastronomía (IAR), C.C. No. 5, 1894 Villa Elisa, Argentina \\ 2 Facultad de Ciencias Astronómicas y Geofísica Universidad Nacional de La Plata, Paseo del Bosque s/n, \\ 1900 La Plata, Argentina \\ ${ }^{3}$ Departamento de Astronomía, Universidad de Chile, Casilla 36-D, Santiago, Chile
}

Received 19 May 2003 / Accepted 8 September 2003

\begin{abstract}
Intermediate angular resolution $\left(H P B W=8\right.$.7) carbon monoxide $\left({ }^{12} \mathrm{CO} J=1 \rightarrow 0\right)$ line observations towards the $\mathrm{OB}$ association Ara OB1 are reported. The molecular line observations cover an area of $2.75 \times 3^{\circ} .00$, and disclose a rich and complex distribution of the molecular line emission. The molecular features likely to be associated with the OB-association Ara OB1 span the velocity range from -28 to $-20 \mathrm{~km} \mathrm{~s}^{-1}$. The most negative radial velocities are observed along NGC 6188 (三Rim nebula). This nebula marks the interface between the HII region RCW 108 and the highly absorbing molecular material located westwards of the ionised region. The dominant $\mathrm{CO}$ structures, labeled $\mathrm{E}$ and $\mathrm{F}$, have a radial velocity of about -23.5 and $-21.8 \mathrm{~km} \mathrm{~s}^{-1}$, respectively. The former harbours a bright optical knot, the infrared cluster RCW-108 and the strong IRAS source $16362-4845$. IRAS point sources are also seen in projection onto ${ }^{12} \mathrm{CO}$ concentrations $\mathrm{F}$ and $\mathrm{H}$. This may indicate that the star forming process is a widespread phenomenon in this molecular complex. Extended continuum emission, having a high degree of spatial correlation with both the infrared and molecular emission, is observed in the region. We believe that this emission, thermal in nature, arises from the ionised surface layers of the molecular complex. The ionising agents are the high mass stars of NGC 6193, the nucleus of Ara OB1. The total amount of molecular gas related to Ara OB1 is about $\sim 1.4 \times 10^{4} M_{\odot}$.
\end{abstract}

Key words. HII regions: individual: RCW 108 - ISM: molecules - open clusters and associations: individual: NGC 6193 open clusters and associations: individual: Ara OB1 - radio continuum: ISM - radio lines: ISM

\section{Introduction}

Ara OB1 is a well defined association of the southern sky covering an area of $\sim 1$ square degree around its central cluster NGC 6193. Whiteoak (1963) determined for this cluster an age of $\sim 5 \times 10^{6} \mathrm{yr}$ and a distance of 1400 pc. NGC 6193 contains two early type stars, HD 150135 and HD 150136, whose spectral types are O7 V and O5: V + O6 (Arnal et al. 1988), respectively. These stars are the main ionizing sources of the low surface brightness optical emission nebula RCW 108 (Rodgers et al. 1960). About $15^{\prime}$ westwards of these stars, there is a bright rim structure that stretches over $30^{\prime}$. This nebula is known as the Rim nebula ( $\equiv$ NGC 6188), and marks the interface between the HII region RCW 108 and a dense nearby dark nebula.

Based on an extensive photometric and photographic UBVRI study, Herbst \& Havlen (1977) derived for Ara OB1 a mean colour excess of $E(B-V)=0.46$ and a distance of $1320 \pm 120$ pc. Comparing the colour-magnitude

Send offprint requests to: E. M. Arnal,

e-mail: arnal@iar .unlp.edu.ar

* Member of the Carrera del Investigador Científico, CONICET, Argentina.

$\star \star$ Fellow from CONICET, Argentina. diagram of Ara OB1 with those of other young clusters, they conclude that Ara OB1 "is about the same age as the Orion nebula cluster $\sim 3 \times 10^{6} y r^{\prime}$. Using $U B V-\mathrm{H} \beta$ photometry a similar colour excess, 0.44 , and distance, $1360 \mathrm{pc}$, were derived by Moffat \& Vogt (1973). Additional distance determinations spans the range between 1100 and $1340 \mathrm{pc}$ (Fitzgerald 1987; Kaltcheva \& Georgiev 1992). Most of the distance determinations favor a value of about $\sim 1300 \mathrm{pc}$.

From spectroscopic observations of probable members of NGC 6193, an average baricentral radial velocity of $-30.0 \pm 2.8 \mathrm{~km} \mathrm{~s}^{-1}$ was determined (Arnal et al. 1988). All velocities in this paper are referred to the Local Standard of Rest. The radial velocity of RCW 108 is about $-20 \mathrm{~km} \mathrm{~s}^{-1}$ (Georgelin \& Georgelin 1970; Cersósimo 1988; Georgelin et al. 1996). A small molecular cloud at a radial velocity of $-17 \mathrm{~km} \mathrm{~s}^{-1}$ (Phillips et al. 1986) was observed near HD 150135/6. They interpreted this cloud as "probably being placental material".

Embedded in a dark cloud located westwards of NGC 6193 there is an $R$ association called Ara R1 (Herbst 1975) and a bright optical knot (Frogel \& Persson 1974). Near infrared and far infrared data revealed the existence of a compact young 


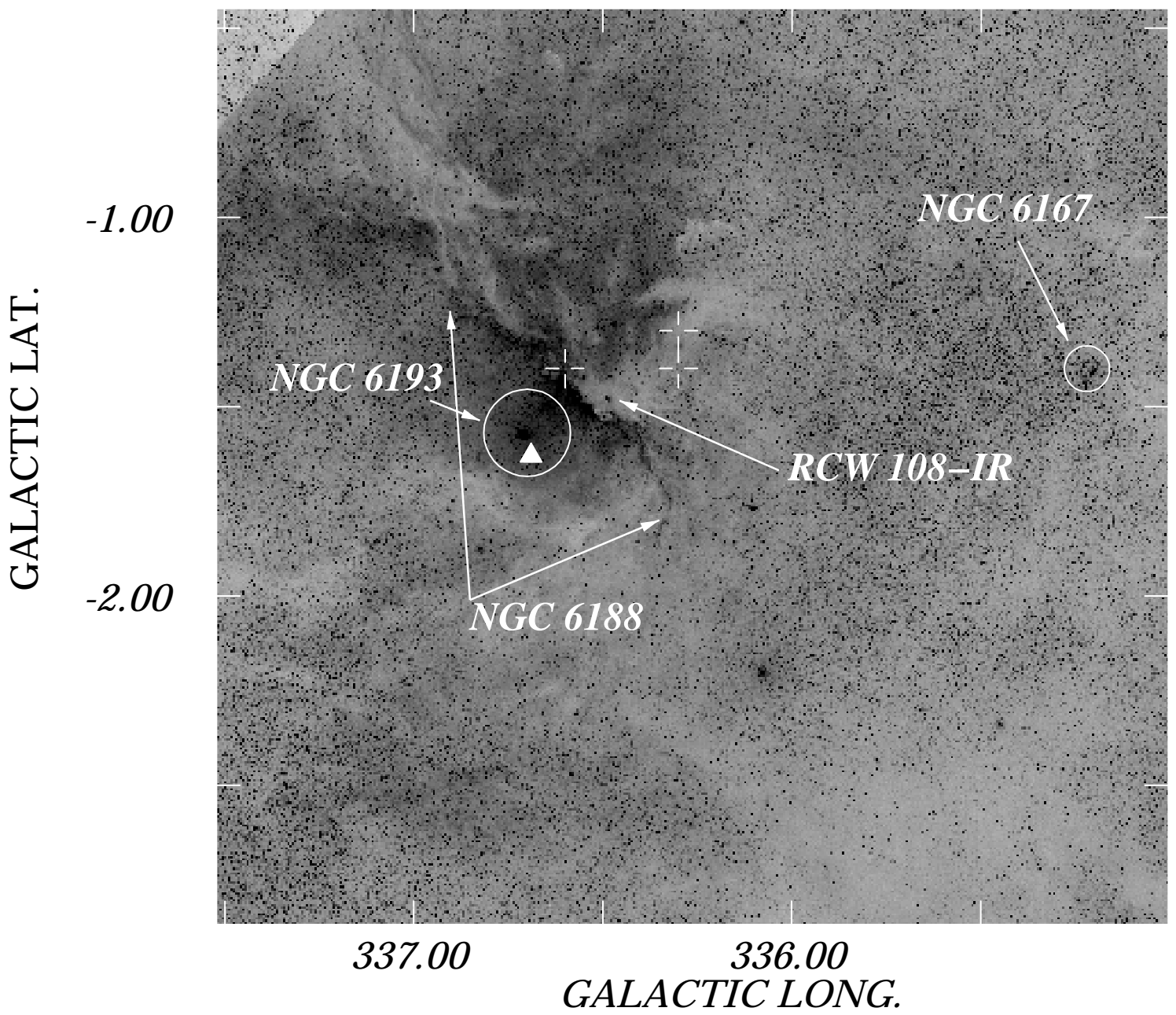

Fig. 1. Grey scale DSS2 R-image of the Ara OB1 region. A light grey tonality indicates a region of high optical obscuration. The diameter of the circles correspond to the optical diameter of the open clusters. The filled in triangle within the circle defining the location of NGC 6193 marks the region observed by Phillips et al. (1986). The plus signs indicate the position of some members of Ara R1 (Herbst 1975). Single-pointing molecular line observations were mostly taken at the position of RCW 108-IR.

cluster, named RCW 108-IR, related to the bright optical knot (Straw et al. 1987).

For the sake of clarity the main optical constituents and their relative location are shown in Fig. 1 superimposed on a red image of the Ara OB1 region retrieved from the Second Generation Digitized Sky Survey (DSS2).

High resolution radio continuum observations at $5 \mathrm{GHz}$ (Goss \& Shaver 1970) detect a small, high emission measure compact HII region slightly eastwards from the bright optical knot. Radio recombination line observations of this source (Wilson et al. 1970; Caswell \& Haynes 1987) provide a radial velocity of about $-25 \mathrm{~km} \mathrm{~s}^{-1}$, that is in very good agreement with molecular line observations of a nearby molecular cloud (OH absorption at $1667 \mathrm{MHz}$ (Caswell \& Robinson 1974), $\mathrm{H}_{2} \mathrm{CO}$ absorption (Whiteoak \& Gardner 1974) and CO emission at $115 \mathrm{GHz}$ (Gillespie et al. 1977; Whiteoak \& Otrupcek 1982)). For all these observations the radial velocity has an average value of $-24 \mathrm{~km} \mathrm{~s}^{-1}$. These molecular line data are single-pointing observations and provide no information on the large scale distribution and kinematics of the molecular gas. Extensive ${ }^{13} \mathrm{CO}(J=1 \rightarrow 0)$ observations of the Ara OB1 region were performed by Yamaguchi et al.(1999), as part of their molecular line studies toward southern sky HII regions "associated with bright rimmed clouds". Though they covered an area large enough to study the large scale distribution of the molecular gas, they focused their research on the association of IRAS point sources with the molecular concentrations found in the area.

Arnal et al. (1987) have found evidence for the presence of an expanding shell of neutral hydrogen in the neighborhoud of the Ara OB1 association. They suggest that this shell, possibly originated by the winds from massive stars of NGC 6167, could have triggered the formation of Ara OB1. Rizzo \& Bajaja (1994) also found evidence for an expanding HI structure in the region. The latter and a multicolor polarimetric study of the region (Waldhausen et al. 1999) are also in line with the sequential star formation scenario suggested by Arnal et al. (1987).

In this paper, using low angular resolution observations $(H P B W=8 ! 7)$ of ${ }^{12} \mathrm{CO}(J=1 \rightarrow 0)$, we carry out the first detailed study of the large scale distribution and kinematics of the molecular gas towards a large region, $334^{\circ} .75 \leq l \leq 337^{\circ} .50$ and $-3.0 \leq b \leq 0.0$, engulfing the association Ara OB 1 . 


\section{Observation and data reduction}

The ${ }^{12} \mathrm{CO}(J=1 \rightarrow 0)$ line observations at 2.6-mm were carried out in November 1996 using the 1.2-m Columbia U. de Chile radiotelescope installed at Cerro Tololo Inter American Observatory (CTIO).

The half-power beam width of the telescope at $115 \mathrm{GHz}$ is 8.7. The receiver front-end was a Schottky barrier diode mixer and a GaAs field-effect transistor amplifier cooled to $77 \mathrm{~K}$ by liquid nitrogen. The typical system temperature, excluding atmospheric contribution, was $370 \mathrm{~K}$ (SSB). A total of 240 positions were observed covering an area of $2.75 \times 3^{\circ}$ in galactic coordinates. The integration time per point vary between 7 and 10 min, depending on source altitude and atmospheric opacity. A 256-channel backend was used as a spectrum analyzer. The observing bandwidth was centred at a radial velocity of $-20 \mathrm{~km} \mathrm{~s}^{-1}$, providing a velocity resolution of $0.26 \mathrm{~km} \mathrm{~s}^{-1}$ and a velocity coverage of $\sim 66 \mathrm{~km} \mathrm{~s}^{-1}$. A second order degree polynomial was substracted from the observations to account for instrumental baseline effects. A typical rms noise per profile was $0.13 \mathrm{~K}$ at $0.26 \mathrm{~km} \mathrm{~s}^{-1}$. Spectra were intensity calibrated individually against a blackbody reference by the standard chopper-wheel method (e.g. Kutner \& Ulrich 1981 and references therein), yielding a temperature scale $T_{\mathrm{a}}^{*}$ corrected for atmospheric attenuation, resistive losses, and rearward spillover and scattering. This radiotelescope has a main beam efficiency of 0.82 (Bronfman et al. 1988).

We also examined continuum data of this region obtained at 1420 and $2417 \mathrm{MHz}$. These data were extracted from a survey carried out with one of the 30-m dishes (Reich et al. 2001) of the Instituto Argentino de Radioastronomía (IAR) and the Parkes 64-m telescope (Duncan et al. 1996), respectively.

In order to analyze the dust distribution, IRAS images at 60 and $100 \mu \mathrm{m}$ were retrieved from the web using the SkyView facility.

\section{Observational results and discussion}

\subsection{Carbon monoxide large scale distribution}

In Fig. 2 a series of four $\mathrm{CO}$ profiles representing mean emission profiles toward different areas around Ara OB1 are shown. Carbon monoxide emission is detected along four main velocity ranges, namely: i) -50 to $-34 \mathrm{~km} \mathrm{~s}^{-1}$; ii) -27 to $-15 \mathrm{~km} \mathrm{~s}^{-1}$; iii) -11 to $-4 \mathrm{~km} \mathrm{~s}^{-1}$; and iv) 0 to $+6 \mathrm{~km} \mathrm{~s}^{-1}$. Figure 2 also shows that the component peaking around $-20 \pm 4 \mathrm{~km} \mathrm{~s}^{-1}$ is the only one detected all over the surveyed area. The peak velocity of this spectral feature varies from $-24 \mathrm{~km} \mathrm{~s}^{-1}$ to $-17 \mathrm{~km} \mathrm{~s}^{-1}$, across the region. In Fig. 3 longitude-latitude images of the $\mathrm{CO}$ emission distribution along the four velocity ranges mentioned above are shown. The area covered by the HII region RCW 108 is depicted by a circumference, while the position of HD $150135 / 6$ is marked by a cross.

It is readily noticeable that the molecular gas distribution is quite dissimilar among the four ranges. In the first one, the bulk of the molecular emission is concentrated toward the galactic plane $(|b| \leq 1.5)$. Within a circle of radius 13.5 centred on HD 150135/6 there is almost no $\mathrm{CO}$ emission above a $5 \sigma$ level.
Moreover, the $\sim 30^{\prime}$ long heavily obscuring dust lane seen in optical images to delimit the west boundary of the Rim nebula, has no counterpart at these velocities. Very likely, molecular gas emission in this velocity range results from large scale galactic structure features (Bronfman et al. 1989).

In the second velocity range, -27 to $-15 \mathrm{~km} \mathrm{~s}^{-1}$, three strong CO features are observed in the vicinity of Ara OB1. Their radial velocity is similar, about $-25 \mathrm{~km} \mathrm{~s}^{-1}$, to those observed in previous molecular line observations. The bright optical knot and the strong infrared source discovered by Frogel \& Persson (1974) are projected onto the molecular concentration peaking at $(l, b)=(336.47,-1.50)$. Furthermore, a low intensity bridge of $\mathrm{CO}$ emission is seen projected onto the region occupied by the dark cloud adjacent to the Rim nebula. Besides the above features, molecular emission extends towards lower galactic latitudes to form a broad ridge that stretches, at a galactic latitude of $b \simeq-2^{\circ} .7$, from $334^{\circ} .9$ to $336^{\circ} .5$ in galactic longitude.

Along the third velocity range, -11 to $-4 \mathrm{~km} \mathrm{~s}^{-1}$, the $\mathrm{CO}$ emission is mostly confined to an ovoidal region delimited by $336^{\circ} .1 \leq l \leq 337.7$ and $-2.5 \leq b \leq-1.5$. Using the galactic rotation model of Brand \& Blitz (1993) (assuming $R_{0}=8.5 \mathrm{kpc}$ and $\Theta_{0}=220 \mathrm{~km} \mathrm{~s}^{-1}$ ) and using an analytical fit to the galactic rotation curve, gas in this velocity range should be located at $0.6 \pm 0.2 \mathrm{kpc}$ from the Sun. Therefore, molecular gas emitting at these velocities very likely is unrelated to Ara OB1.

In the last velocity range, 0 to $+6 \mathrm{~km} \mathrm{~s}^{-1}$, there is almost no $\mathrm{CO}$ emission above $5 \sigma$ level within $\sim 1^{\circ} .03$ from Ara OB 1 . The only exception to this is a small feature observed at $(l, b)=$ (336.01, -1.63). The molecular emission is mostly confined to the lower right part of the image, reaching a maximum of $\sim 0.8 \mathrm{~K}$ around $(l, b)=\left(335^{\circ} .5,-2^{\circ} .0\right)$. According to circular galactic rotation models, this gas at positive velocities should be placed beyond the solar circle, at a distance of $\sim 16 \mathrm{kpc}$.

Based on radial velocity arguments and the spatial location of the main molecular concentrations with respect to Ara OB1, it is very likely that most of the molecular gas probably associated with the stellar association is present in the velocity range from -27 to $-15 \mathrm{~km} \mathrm{~s}^{-1}$. In order to follow in more detail its spatial distribution, in Fig. 4 a mosaic of ten images is shown. These images cover the velocity range from -31.1 to $-10.2 \mathrm{~km} \mathrm{~s}^{-1}$, and every image represents a mean of the emission over a velocity interval of $\sim 2.1 \mathrm{~km} \mathrm{~s}^{-1}$. The central velocity of each image is indicated in its inner lower left corner. To help characterize the large scale velocity field observed in the velocity range from -28 to $-13 \mathrm{~km} \mathrm{~s}^{-1}$, different $\mathrm{CO}$ concentrations are identified in Fig. 4. They are arranged in order of increasing radial velocity, and are labelled from A through P in Fig. 4. These narrow velocity channels show that the molecular gas distribution changes quite dramatically as we move along this velocity range. From a general point of view these images show that: $a$ ) the dark cloud adjacent to the Rim nebula, represented in the first two maps of Fig. 4 by a thick line, has a counterpart only along a narrow velocity range centered at $-27 \mathrm{~km} \mathrm{~s}^{-1}$ (Features A, B and $\mathrm{D}) ; b) \mathrm{CO}$ emission close $\left(\leq 10^{\prime}\right)$ to Ara OB1 begins to be noticeable about $-30 \mathrm{~km} \mathrm{~s}^{-1}$; $c$ ) the dominant $\mathrm{CO}$ structures depicted in Fig. $3 \mathrm{~b}$ are observed along different velocity 

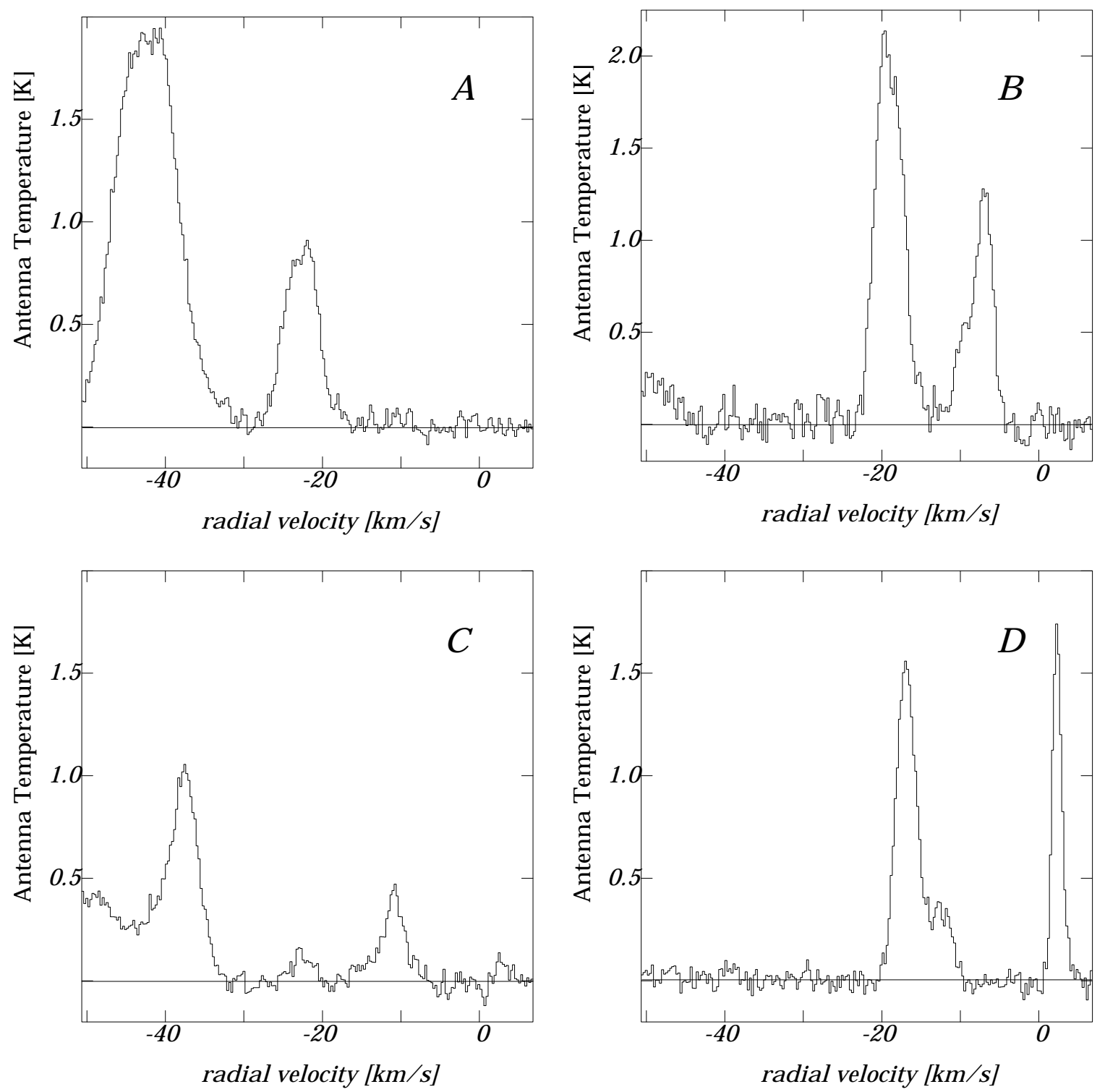

Fig. 2. Representative sample of average $\mathrm{CO}$ profiles obtained toward different regions of the Ara OB1 complex. The profile units are $T_{\mathrm{A}}^{*}$ in $\mathrm{K}$ (ordinate) and $V_{\mathrm{LSR}}$ in $\mathrm{km} \mathrm{s}^{-1}$ (abscissa).

ranges. The structure peaking at $(l, b)=(336.47,-1.50)($ see Fig. 3b) becomes first noticeable about $-29 \mathrm{~km} \mathrm{~s}^{-1}$, achieves its peak temperature at $-24 \mathrm{~km} \mathrm{~s}^{-1}$ and is barely detectable beyond $-17 \mathrm{~km} \mathrm{~s}^{-1}$ (Feature E). On the other hand, the structure at $(l, b)=(336.25,-1.25)$ is first visible as a distortion of the low emission levels at $-26 \mathrm{~km} \mathrm{~s}^{-1}$, becomes the brightest feature present in the image at $-22 \mathrm{~km} \mathrm{~s}^{-1}$ and fades away at $-17 \mathrm{~km} \mathrm{~s}^{-1}$ (Feature F). Lastly, the object seen at $(l, b)=\left(336.60,-1^{\circ} .77\right)$ appears at more positive velocities than the other features. It becomes detectable at a $5 \sigma$ level at $-24 \mathrm{~km} \mathrm{~s}^{-1}$, peaks at $-20 \mathrm{~km} \mathrm{~s}^{-1}$ and is hardly visible beyond $-16 \mathrm{~km} \mathrm{~s}^{-1}$ (Feature $\mathrm{H}$ ); $d$ ) the broad ridge of $\mathrm{CO}$ emission shown in Fig. $3 \mathrm{~b}$ as a series of scatter maxima at $b=-2.7$ and $335^{\circ} .00 \leq l \leq 336.50$, is first detected at $-20 \mathrm{~km} \mathrm{~s}^{-1}$ as a low latitude extent of the feature peaking at $(l, b)=(336.60$, $-1.77)$, achieves its maximum latitude extent at $-18 \mathrm{~km} \mathrm{~s}^{-1}$, and only its low longitude extreme $\left(l \leq 336^{\circ} .00\right)$ remains visible beyond $-16 \mathrm{~km} \mathrm{~s}^{-1}$ (Features J, K, L, M and N).
The main parameters of the structures found above are given in Table 1. The quoted position for a given $\mathrm{CO}$ concentration is the one corresponding to its peak emission. Bearing in mind the spatial distribution shown in Fig. 3, it is very likely that more than one spectral feature will be present at a given spatial location in Fig. 4. The number of spectral features present in the spectrum is given in the second column of Table 1 . The peak temperature, radial velocity and linewidth of the different components were derived from a Gaussian fitting.

\subsection{Infrared data}

\subsubsection{Extended emission}

The $60 \mu \mathrm{m}$ and $100 \mu \mathrm{m}$ IRAS band maps of the region were downloaded using the SkyView facility. In Fig. 5 the $60 \mu \mathrm{m}$ IR emission map superimposed on the $\mathrm{CO}$ emission map observed in the velocity range -25 to $-21 \mathrm{~km} \mathrm{~s}^{-1}$ is shown. Most 

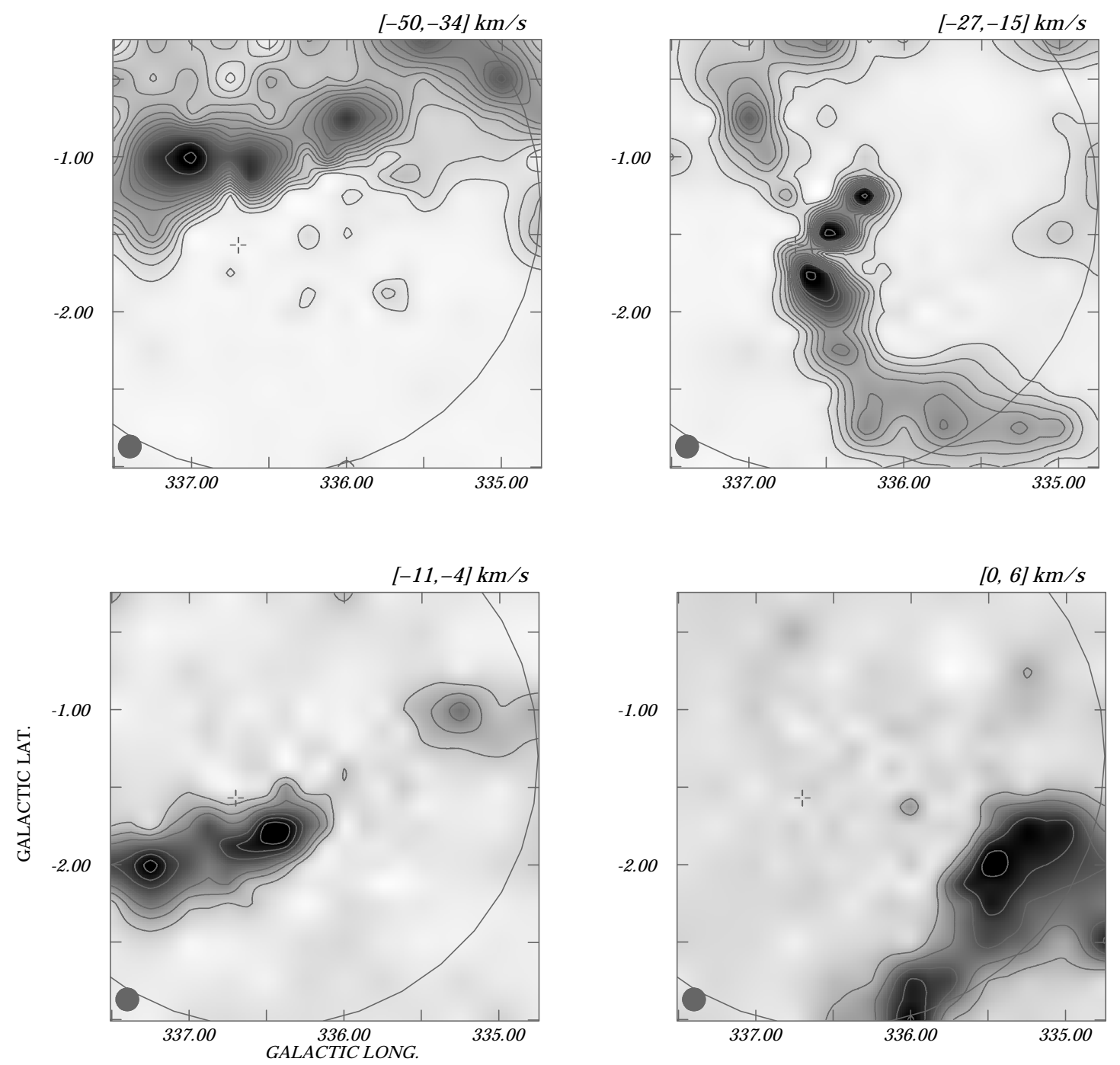

Fig. 3. Images of the spatial distribution of the $\mathrm{CO}$ emission in four different velocity ranges. The velocity range is given in the upper right corner of each figure. The large circle indicates the angular extent of RCW 108. The beam size of the CO observations is shown by a shaded circle in the lower left corner of each map. The position of HD 150135/6 is marked by a plus sign. Low CO emission regions are indicated by a light grey tonality. The lowest temperature contour and the contour spacing is $0.19 \mathrm{~K}(11 \sigma)$. The minimum and maximum grey-scale values are -0.15 and $3.4 \mathrm{~K}$ for the upper left panel, -0.15 and $2.71 \mathrm{~K}$ for the upper right panel, -0.15 and $1.36 \mathrm{~K}$ for the lower left panel, and -0.15 and $0.96 \mathrm{~K}$ for the lower right panel.

of the IR emission is concentrated towards the galactic plane $\left(b \leq \mid 1^{\circ}\right)$, but at $l \simeq 336.5$ there is an extended plateau of emission that can be traced down to $b \sim-2^{\circ}$.0. Superimposed on this plateau three IR concentrations having an excellent spatial correlation with $\mathrm{CO}$ features labeled $\mathrm{E}, \mathrm{F}$ and $\mathrm{H}$ are easily noticeable. The IR feature closer to NGC 6193 is the strongest one. A characteristic seen in all three concentrations is that there is a slight offset in position between the location of both the IR and CO peaks, in the sense that along a hypothetical line joining NGC 6193 and the peaks of the CO concentrations, the IR peaks are always closer to Ara OB1 than the molecular ones. A weak IR emission seen towards the CO feature D may signal infrared emission arising from the dusty interface between RCW 108 and the nearby molecular material.

In order to derive some characteristic physical parameters of the three main infrared features, their fluxes at 60 and $100 \mu \mathrm{m}$ were computed, after removing a local emission background, and are given in Table 2 . The coordinates of the features are given in the first and second columns. The source dimensions, corrected for beam broadening, in galactic longitude and latitude and the position angle of the major axis, are given in the third, fourth, and fifth columns, respectively. The fluxes at 60 and $100 \mu \mathrm{m}$ are given in the next two columns, whilst an estimate of the mean dust temperature, $T_{\mathrm{d}}$, is provided in the last two columns. The latter is derived by fitting to the data a modified Planck function of the form $v^{m} \times B_{v}\left(T_{\mathrm{d}}\right)$, where the first term accounts for the frequency dependence of the grain emissivity. Two values of $T_{\mathrm{d}}$ for $m=2$ and for $m=1.5$ (given in bracketts) are listed. In the last column a capital letter identifies the IR feature with its CO counterpart (see Table 1). The quoted source peak coordinates and angular extent were derived from a bidimensional Gaussian fitting to the IR distribution at $60 \mu \mathrm{m}$. Those values derived from the $100 \mu \mathrm{m}$ map are almost identical. The 60 and $100 \mu \mathrm{m}$ fluxes were 

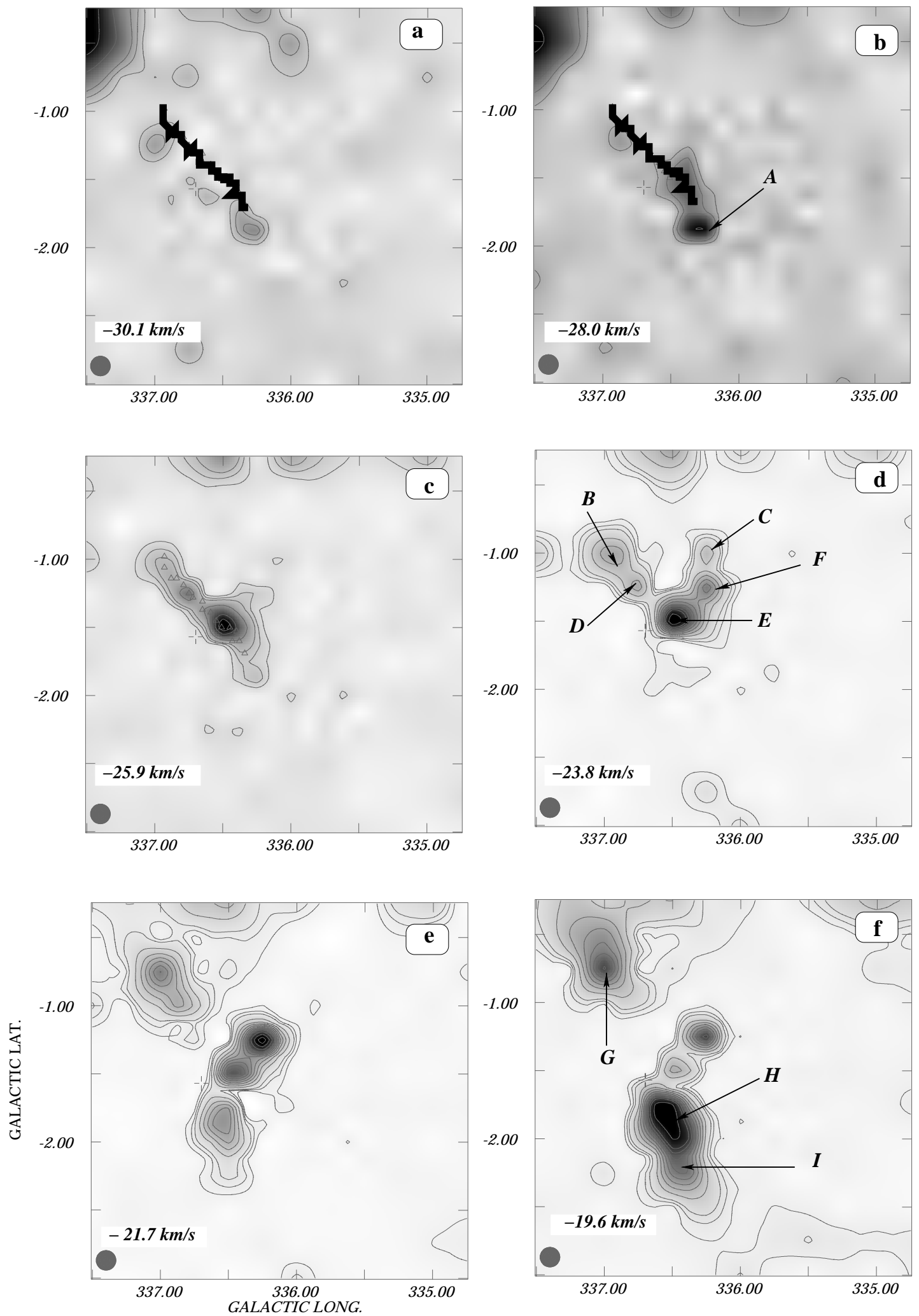

Fig. 4. Distribution of the $\mathrm{CO}$ emission for selected velocity ranges. The velocity coverage of each map is $2.1 \mathrm{~km} \mathrm{~s}^{-1}$ and its central velocity is given by the number printed in each panel around $\left(337^{\circ} .00,-2^{\circ} .60\right)$. The beam size of the CO observations is shown by a shaded circle in the lower left corner of each map. The position of HD 150135/6 is marked by a plus sign. Low CO emission regions are indicated by a light grey tonality. The lowest temperature contour $0.19 \mathrm{~K}(5 \sigma)$. The contour spacing is $0.19 \mathrm{~K}$ till $0.57 \mathrm{~K}, 0.57 \mathrm{~K}(15 \sigma)$ between 1.14 and $5.13 \mathrm{~K}$ and $1.14 \mathrm{~K}(30 \sigma)$ between 6.27 and $8.55 \mathrm{~K}$. The minimum grey-scale value is always $-0.15 \mathrm{~K}$ whilst the maximum grey-scale value is 1.19 , $3.01,6.54,8.38,6.60,6.15,3.74,2.33$ and $3.45 \mathrm{~K}$ for panels a), b), c), d), e), f), g), h), i) and j), respectively. In panels a) and b) the location of NGC 6188 (三the Rim nebula) is indicated by a thick broken line. The capital letters identify the structures listed in Table 1. 
1.00
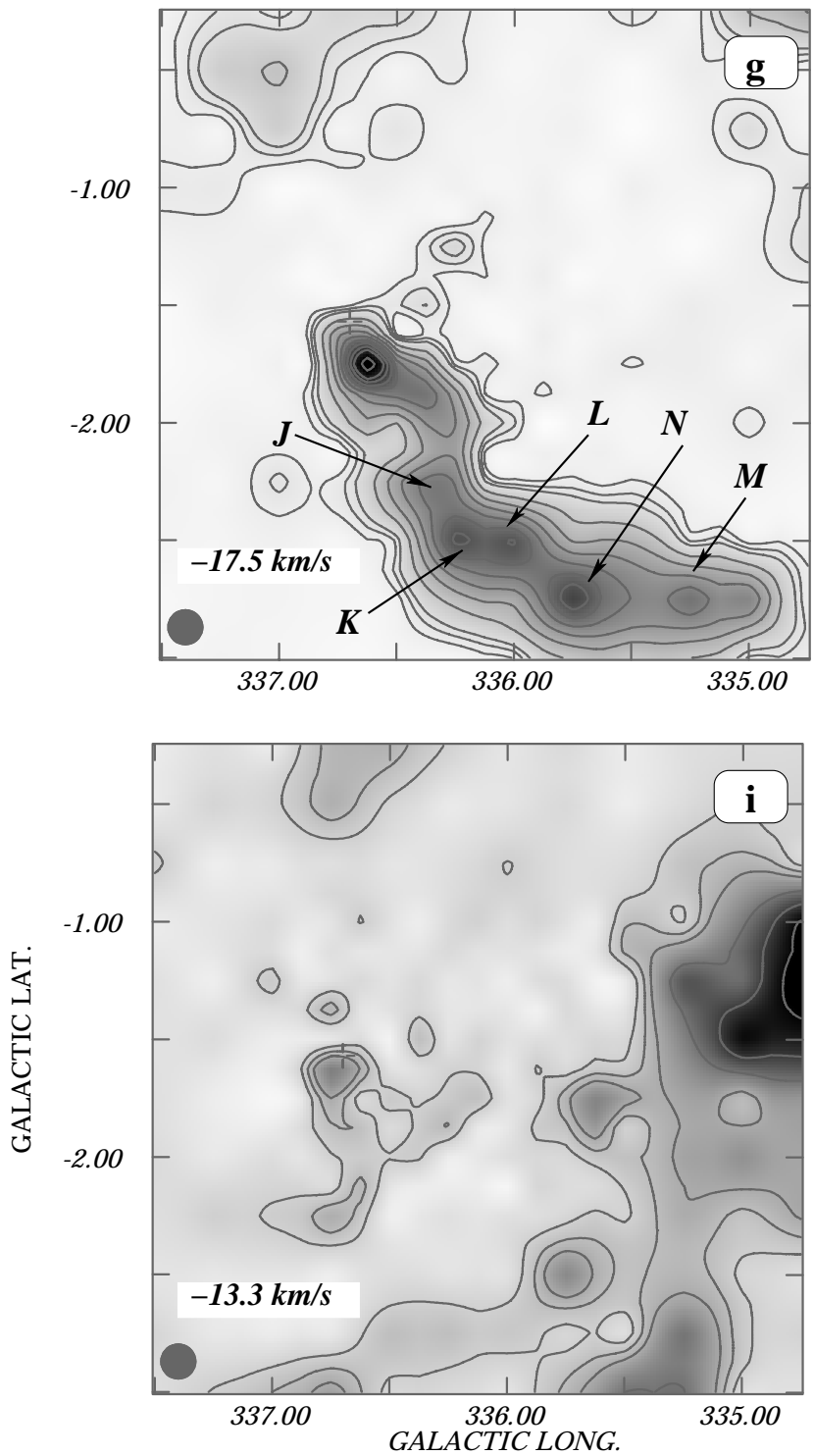

Fig. 4. continued.

measured in two ways, firstly by fitting a two-dimensional Gaussian and secondly by integrating over different polygons surrounding the sources. In all cases a twisted-plane background was substracted off. In this way for sources D and E, at both 60 and $100 \mu \mathrm{m}$, fluxes are consistent with each other to $4 \%$, while for source $\mathrm{F}$ different methods agree to $9 \%$ (at $60 \mu \mathrm{m}$ ) and 25\% (at $100 \mu \mathrm{m}$ ), respectively. In Table 2 a mean value for the different flux determinations is given. These uncertainties and those given by Fich \& Terebey (1996) were used to derived the dust temperature uncertainties quoted in Table 2. Based on these estimates, there is a slight hint that the dust concentration coinciding in the plane of the sky with the $\mathrm{CO}$ feature $\mathrm{E}$, the closest to Ara OB1, may be warmer than those IR features seen towards $\mathrm{CO}$ features $\mathrm{F}$ and $\mathrm{H}$, respectively.

\subsubsection{Point sources}

Thermal emission from dust grains may also pinpoint the presence of cold protostars or circumstellar disks and envelopes
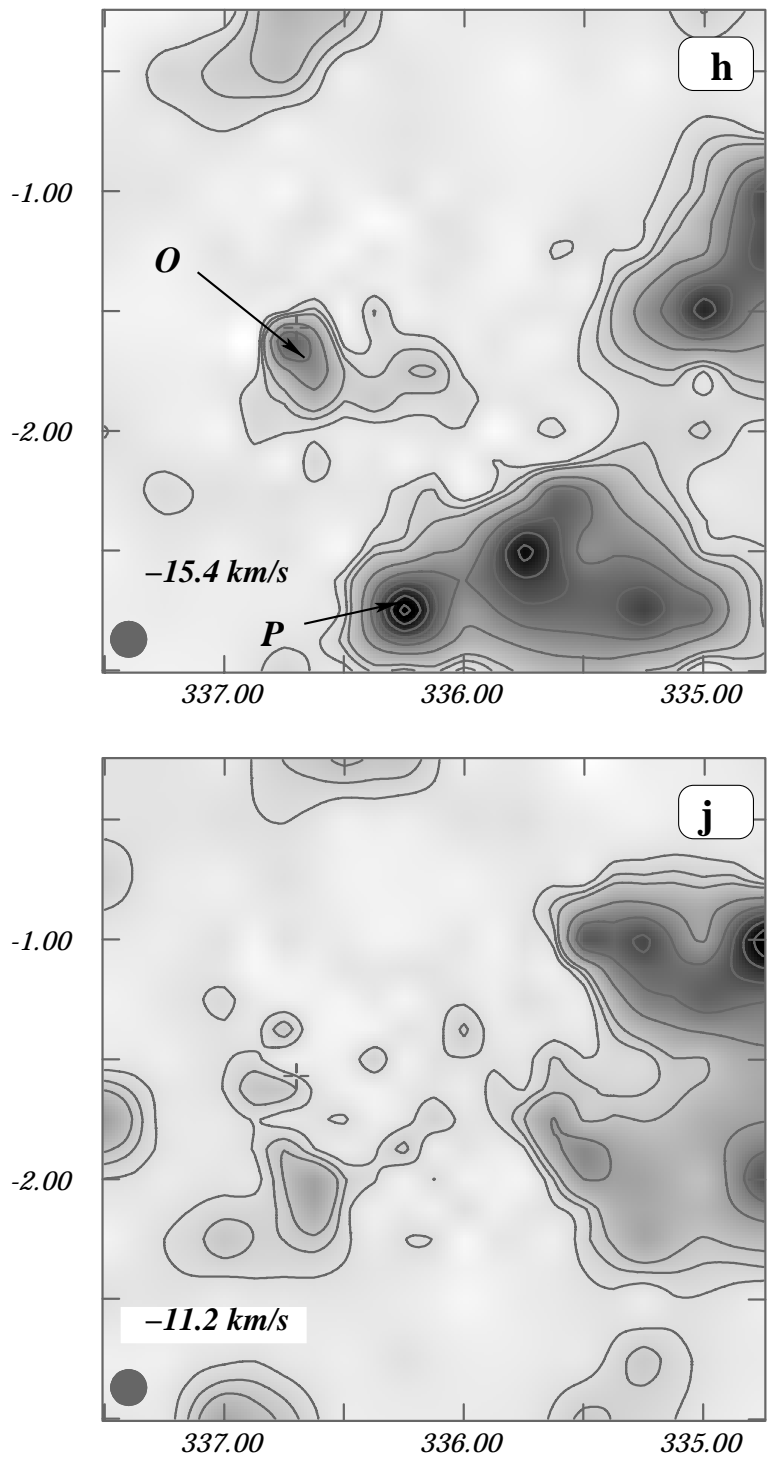

around young stellar objects (Henning 1996). Arnal et al. (1987) have found evidence for an expanding shell of neutral and molecular gas coexisting with the $\mathrm{OB}$ association. They suggested that this shell could have triggered the formation of Ara OB1. In an attempt to look for representatives of a younger generation of stars that may still be deeply buried in the molecular clouds observed towards Ara OB1, we have selected IRAS point sources that are located within the limits of a circular region, of radius $35^{\prime}$, centered at $(l, b)=(336.5,-1.5)$. A total of 87 sources were found. Applying the selection criteria used by Junkes et al. (1992), namely: i) $F_{100} \leq 100 \mathrm{Jy}$; ii) $1.2 \leq F_{100} / F_{60} \leq 6.0$; iii) $F_{25} \leq F_{60}$; and $\left.i v\right) Q_{60}+Q_{100} \geq 4$, where $F_{\lambda}$ is the IRAS flux density at wavelength $\lambda$, and $Q_{\lambda}$ describes the quality factor of the IRAS fluxes, and requiring the IRAS source to be seen in projection onto a molecular complex, a total of six infrared protostellar candidates were found. These sources are indicated in Table 3 by a letter "J" in the last column. In a recent paper by Yamaguchi et al. (1999), using different selection criteria, they claimed that within the same area nine IRAS sources may be possibly associated with 
Table 1. Position and kinematical data of the main CO features.

\begin{tabular}{|c|c|c|c|c|c|c|}
\hline Feature & $n$ & $\begin{array}{c}l \\
\left({ }^{\circ}\right)\end{array}$ & $\begin{array}{l}b \\
\left({ }^{\circ}\right)\end{array}$ & $\begin{array}{r}T_{\max } \\
(\mathrm{K})\end{array}$ & $\begin{array}{c}V_{\max }^{b} \\
\left(\mathrm{~km} \mathrm{~s}^{-1}\right)\end{array}$ & $\begin{array}{l}F W H M^{b} \\
\left(\mathrm{~km} \mathrm{~s}^{-1}\right)\end{array}$ \\
\hline \multirow[t]{4}{*}{ A } & 4 & 336.38 & -1.88 & 1.0 & -28.6 & 2.7 \\
\hline & & & & 4.0 & -19.0 & 2.4 \\
\hline & & & & 0.9 & -9.9: & 1.2: \\
\hline & & & & 0.8 & $-7.8:$ & 3.0: \\
\hline \multirow[t]{3}{*}{ B } & 3 & 336.94 & -1.00 & 4.8 & $-43.0^{a}$ & $8.7^{a}$ \\
\hline & & & & 2.1 & -24.0 : & 2.9: \\
\hline & & & & 1.6 & $-21.5:$ & 2.2: \\
\hline \multirow[t]{2}{*}{$\mathrm{C}$} & 2 & 336.25 & -1.00 & 2.0 & -45.6 & 3.7 \\
\hline & & & & 2.3 & -23.8 & 1.5 \\
\hline \multirow[t]{2}{*}{ D } & 2 & 336.75 & -1.25 & 1.3 & -40.1 & 2.0 \\
\hline & & & & 2.3 & -24.7 & 3.6 \\
\hline E & 1 & 336.44 & -1.50 & 6.3 & -23.4 & 4.5 \\
\hline $\mathrm{F}$ & 1 & 336.25 & -1.25 & 10.0 & -21.9 & 3.3 \\
\hline \multirow[t]{2}{*}{$\mathrm{G}$} & 2 & 337.00 & -0.75 & 1.2 & $-43.7^{a}$ & $7.0^{a}$ \\
\hline & & & & 7.7 & -20.7 & 2.1 \\
\hline \multirow[t]{2}{*}{$\mathrm{H}$} & 2 & 336.56 & -1.88 & 6.5 & -19.9 & 3.5 \\
\hline & & & & 1.9 & -7.2 & 3.0 \\
\hline I & 1 & 336.50 & -2.13 & 5.0 & -20.1 & 2.4 \\
\hline $\mathbf{J}$ & 1 & 336.38 & -2.25 & 4.4 & -19.1 & 2.4 \\
\hline $\mathrm{K}$ & 1 & 336.25 & -2.50 & 4.9 & -18.1 & 2.2 \\
\hline $\mathrm{L}$ & 1 & 336.00 & -2.50 & 5.8 & -17.5 & 1.8 \\
\hline \multirow[t]{2}{*}{ M } & 2 & 335.75 & -2.50 & 5.8 & -17.5 & 2.4 \\
\hline & & & & 3.8 & $-1.6:$ & 1.0: \\
\hline \multirow[t]{2}{*}{$\mathrm{N}$} & 2 & 335.25 & -2.75 & 3.9 & -17.0 & 3.7 \\
\hline & & & & 1.0 & +2.2 : & 0.6 : \\
\hline $\mathrm{O}$ & 1 & 336.69 & -1.63 & 2.4 & -17.5 & 5.5 \\
\hline \multirow[t]{2}{*}{$\mathrm{P}$} & 2 & 336.25 & -2.75 & 5.5 & -16.3 & 2.0 \\
\hline & & & & 1.3 & +3.8 & 0.8: \\
\hline
\end{tabular}

${ }^{a}$ Blend of several components.

$b$ A colon (:) at the end indicates an unreliable fit.

molecular clouds related to RCW 108. These sources are identified in Table 3 by a letter "Y" in the last column. In Fig. 5 the position of the former are mark by small crosses while the location of the later are indicated by open triangles. In the same figure the large cross marks the position of HD 150135/6.

Though the "class" of an object, exclusively based on its location in an IRAS-based color-color diagram, is far from being determined without ambiguity (e.g. different "classes" of objects may fall within the same occupation zones (see Walker et al.1989)), it is striking that most of the object listed in Table 3 appear projected onto the brightest parts of the molecular concentrations. The brightest infrared source, IRAS 16362-4845, coincides with a compact young infrared cluster of stars (Straw et al. 1987). This cluster may represent "the tip of the iceberg of a larger population of (young) stars"
(Melnick 1992). The IRAS sources given in Table 3 may pinpoint to sites where star formation is taken place throughout the Ara OB1 complex.

\subsection{Radiocontinuum data}

A $2.4 \mathrm{GHz}$ small scale component image (Duncan et al. 1995) of a region larger than the one covered by the Ara OB1 complex is shown in Fig. 6. Noteworthy the continuum emission extendes towards lower galactic latitudes between the galactic longitude interval $336^{\circ} 0 \leq l \leq 338^{\circ} 0$. This feature, that appears as detached from the overall galactic continuum emission that is mostly confined at $|b| \leq 1$. 0 , depicts a strong point-like object superimposed on to an extended emission. From here onwards we shall refer to the latter as to the 


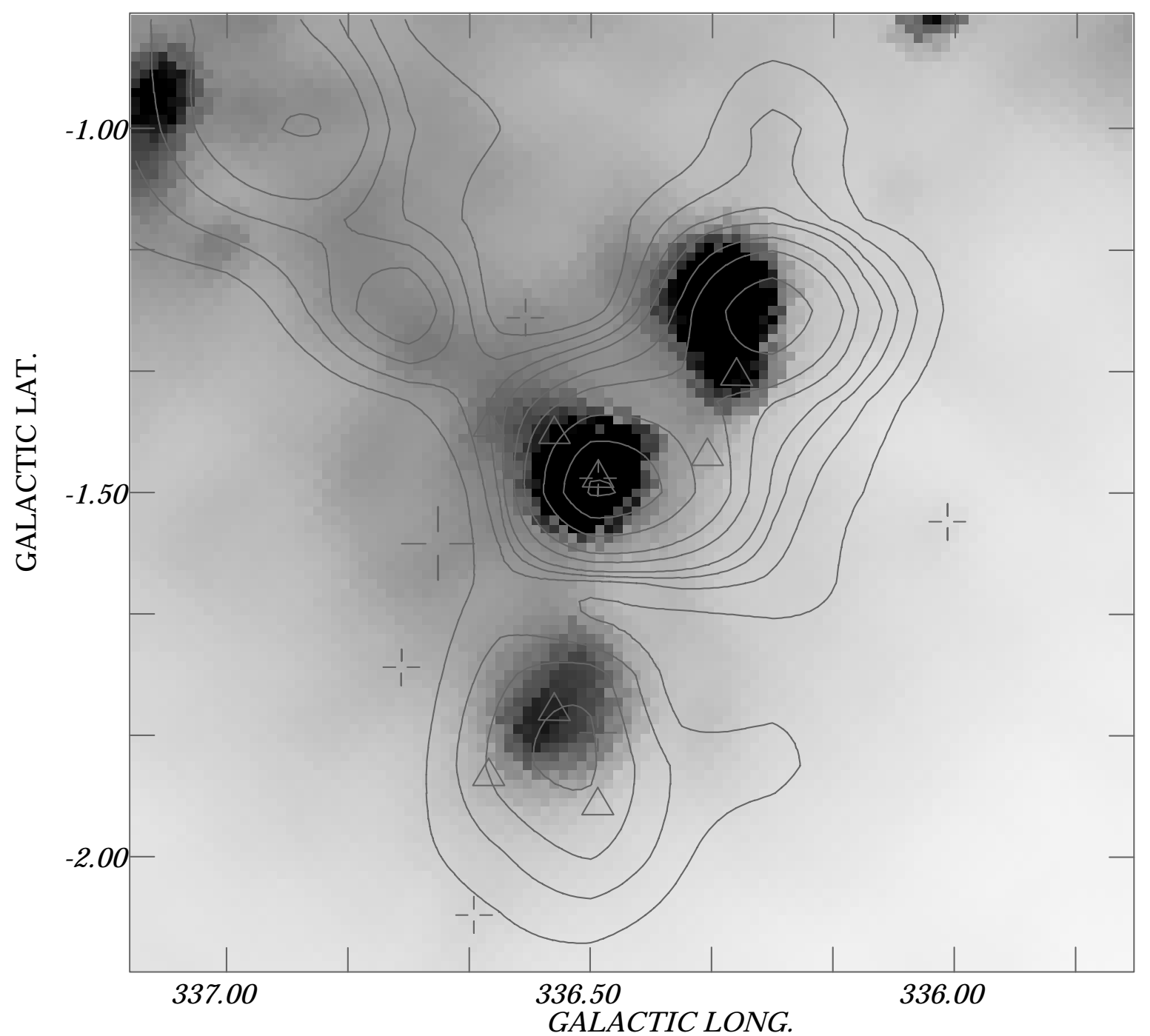

Fig. 5. Overlay of the IRAS $60 \mu \mathrm{m}$ emission (grey scale) and the mean CO emission along the velocity range -25.0 to $-21.0 \mathrm{~km} \mathrm{~s}{ }^{-1}$. The grey scale minimum and maximum are 200 and $750 \mathrm{MJy} / \mathrm{sr}$. The lowest countour level is $0.25 \mathrm{~K}(8 \sigma)$ and the next contour levels are $0.5,0.75,1.0$, 1.5 and $2 \mathrm{~K}$. From there onwards the contour spacing is $1 \mathrm{~K}$. The position of HD 150135/6 is marked by a large cross. The position of those sources fullfilling the Junkes criteria are marked by a small cross, while the location of those having a capital "Y" in the last column of Table 3 is given by an open triangle. Notice the relative shift in position between the infrared and CO maxima.

Table 2. Flux densities at 60 and $100 \mu \mathrm{m}$ and dust parameters.

\begin{tabular}{cccccccccc}
\hline \hline$l$ & $b$ & $\Theta_{l}$ & $\Theta_{b}$ & $\mathrm{PA}$ & $S(60 \mu \mathrm{m})$ & $S(100 \mu \mathrm{m})$ & $T_{\mathrm{d}}(m=1.5)$ & $T_{\mathrm{d}}(m=2.0)$ & Notes \\
$\left({ }^{\circ}\right)$ & $\left({ }^{\circ}\right)$ & $\left({ }^{\prime}\right)$ & $\left({ }^{\prime}\right)$ & $\left({ }^{\circ}\right)$ & $(\mathrm{Jy})$ & $(\mathrm{Jy})$ & $(\mathrm{K})$ & $(\mathrm{K})$ & \\
\hline 336.31 & -1.25 & 7.5 & 5.6 & 2 & 7500 & 12400 & $34.2 \pm 6.8$ & $30.9 \pm 5.6$ & $\mathrm{~F}$ \\
336.49 & -1.48 & 4.5 & 3.6 & 144 & 17000 & 23400 & $37.3 \pm 8.1$ & $33.2 \pm 6.4$ & $\mathrm{E}$ \\
336.54 & -1.79 & 10.4 & 8.0 & 154 & 2900 & 5400 & $32.5 \pm 6.2$ & $29.5 \pm 5.2$ & $\mathrm{H}$ \\
\hline
\end{tabular}

plateau. In Fig. 7 the $2.4 \mathrm{GHz}$ continuum emission and the $\mathrm{CO}$ spatial distribution observed in the velocity range from -25 to $-21 \mathrm{~km} \mathrm{~s}^{-1}$ are shown together. In this image the point-like source coincides with the $\mathrm{CO}$ feature labelled E. Its total flux density is $S_{2.4 \mathrm{GHz}}=9.2 \pm 0.6 \mathrm{Jy}$ and its peak emission is located at $(l, b)=(336.52,-1.48)$. This source very likely represents the $2.4 \mathrm{GHz}$ counterpart of the compact HII region detected at $5 \mathrm{GHz}\left(S_{5 \mathrm{GHz}}=8.6 \mathrm{Jy}\right)$ by Goss \& Shaver (1970), and interferometric observations at $0.408 \mathrm{GHz}\left(S_{0.4 \mathrm{GHz}}=2.2 \mathrm{Jy}\right)$ by Shaver \& Goss (1970). The observed flux densities are fully consistent with a thermal nature for this source.

After removing the point-like source, the plateau continuum emission shows some degree of morphological correspondence with the $\mathrm{CO}$ structures labelled A, B, D, E, H and $\mathrm{O}$. Its total flux density at $2.4 \mathrm{GHz}$ is $210 \pm 23 \mathrm{Jy}$. Under the assumption of an optically thin thermal continuum emission, 
Table 3. IRAS point sources.

\begin{tabular}{rccrrrrc}
\hline \hline IRAS source & $l$ & $b$ & $S(12 \mu \mathrm{m})$ & $S(25 \mu \mathrm{m})$ & $S(60 \mu \mathrm{m})$ & $S(100 \mu \mathrm{m})$ & Notes \\
& $\left({ }^{\circ}\right)$ & $\left({ }^{\circ}\right)$ & $(\mathrm{Jy})$ & $(\mathrm{Jy})$ & $(\mathrm{Jy})$ & $(\mathrm{Jy})$ & \\
\hline $16345-4910$ & 336.01 & -1.54 & 2.5 & 1.9 & 26.4 & 127.0 & $\mathrm{~J}$ \\
$16348-4849$ & 336.30 & -1.34 & 2.7 & 17.1 & 356.0 & 4080.0 & $\mathrm{Y}$ \\
$16355-4851$ & 336.34 & -1.45 & 2.5 & 2.6 & 46.0 & 901.0 & $\mathrm{Y}$ \\
$16381-4912$ & 336.37 & -1.99 & 0.7 & 1.4 & 25.6 & 311.0 & $\mathrm{Y}$ \\
$16362-4845$ & 336.49 & -1.48 & 208.0 & 2980.0 & 12200.0 & 18700.0 & $\mathrm{~J}, \mathrm{Y}$ \\
$16378-4900$ & 336.49 & -1.83 & 2.0 & 1.1 & 76.8 & 435.0 & $\mathrm{~J}$ \\
$16382-4904$ & 336.49 & -1.93 & 1.2 & 1.3 & 26.5 & 399.0 & $\mathrm{Y}$ \\
$16362-4841$ & 336.55 & -1.42 & 4.9 & 12.3 & 77.0 & 18700.0 & $\mathrm{Y}$ \\
$16379-4856$ & 336.55 & -1.80 & 2.6 & 17.9 & 164.0 & 516.0 & $\mathrm{Y}$ \\
$16357-4832$ & 336.59 & -1.26 & 1.4 & 2.9 & 40.7 & 63.9 & $\mathrm{~J}$ \\
$16365-4836$ & 336.64 & -1.41 & 1.6 & 2.3 & 48.5 & 138.0 & $\mathrm{Y}$ \\
$16387-4855$ & 336.64 & -1.89 & 0.8 & 3.1 & 13.6 & 339.0 & $\mathrm{Y}$ \\
$16385-4844$ & 336.76 & -1.74 & 1.4 & 0.8 & 8.5 & 49.6 & $\mathrm{~J}$ \\
$16396-4902$ & 336.66 & -2.08 & 0.9 & 1.3 & 13.8 & 81.6 & $\mathrm{~J}$ \\
\hline
\end{tabular}

the total number of ionising photons needed to account for the continuum flux is $N_{\text {Lyc }} \simeq(3.0 \pm 0.3) \times 10^{49} \mathrm{~s}^{-1}$ (Chaisson 1976). Bearing in mind possible geometrical effects (e.g.: the relative location of NGC 6193 with respect to the nearby molecular clouds), adopting for the most massive stars of this open cluster the spectral types provided by Arnal et al. (1987), and relying on the ionising fluxes calculated by Schaerer \& de Koter (1997), the most massive stars of this open cluster are quite capable of providing the required number of Lyman continuum photons.

In this context, we interpret the plateau continuum emission as mainly arising from the ionized skin of those molecular clouds located in the vicinity of NGC 6193. On the other hand, the surface layers of those $\mathrm{CO}$ structures located further away from NGC 6193 (e.g.: CO features C and F) should be hit by a smaller number of ionizing photons, thus explaining the weakness (or lack of) continuum emission possibly arising from these molecular concentrations.

Summing up, the point-like continuum source very likely represents a region of on going star formation within the Ara OB1 complex, while the plateau emission may be originated in the ionized surface layers of those molecular clouds located in the neighbourhood of NGC 6193.

\subsection{Molecular gas associated with AraOB1}

In Fig. 8 an overlay between a MAMA R-image of the region and the integrated molecular emission in four velocity spanning the interval from -27.1 to $-20.1 \mathrm{~km} \mathrm{~s}^{-1}$ is shown. The position of the most conspicuous members of NGC 6193 (HD 150135/6) is marked by a cross. In the optical image the interface between the HII region RCW 108 and nearby (to NGC 6193) molecular clouds is delinated by a dark grey lane running diagonally, at a position angle of $\sim 46^{\circ}$, from $(l, b)=\left(336^{\circ} .41,-1.58\right)$ till $\left(336^{\circ} .92,-1.03\right)$. It is worth mentioning that in all these images the molecular emission has a very good spatial correlation with the high obscuration regions (light grey tones in the optical image) seen in the neighbourhood of Ara OB1.

The peaks of $\mathrm{CO}$ emission are always seen projected, in the velocity range from -27.1 to $-25.5 \mathrm{~km} \mathrm{~s}^{-1}$ (see Fig. 8a) along the high extinction lane running paralell to the ridge of high intensity $\mathrm{H}_{\alpha}$ emission that signals the interface between RCW 108 and a nearby molecular cloud. The maximum of the $\mathrm{CO}$ emission at $(l, b)=(336.44,-1.50)$ is coincident with the location of the bright optical knot and the strong infrared source discovered by Frogel \& Persson (1974) (RCW 108-IR in Fig. 1). Towards lower galactic latitudes the low level emission abruptly changes in direction at $(l, b)=\left(336^{\circ} .40,-1^{\circ} .55\right)$. A closer look at the optical image reveals that this sudden bend closely follows a part of the Rim Nebula that in this region is not so bright at optical wavelengths as the one seen in the immediate vicinity of NGC 6193. Based on above, we interpret the bulk of the low level emission seen in this velocity range as arising from molecular gas related to those dark clouds which define the low longitude boundary of NGC 6188 (三Rim Nebula). All the single-pointing molecular line observations carried out in the past, were taken at the position where the brightest $\mathrm{CO}$ emission is observed (Feature E in Fig. 4). Bearing in mind the agreement in velocity between our $\mathrm{CO}$ data and other gaseous tracers (e.g. radio recombination lines and other molecular species) and the excellent morphological correlation between feature $\mathrm{E}$ and an optically visible dark cloud, a close relationship between this feature and the Ara OB1 complex is very likely.

At slightly more positive velocities, -25.3 to $-23.7 \mathrm{~km} \mathrm{~s}^{-1}$ (see Fig. 8b), the above mentioned pattern still persists, but two new molecular concentrations are observed westwards of feature $\mathrm{E}$. These features, labelled $\mathrm{C}$ and $\mathrm{F}$, also have a quite striking morphological correspondence with high obscuration 


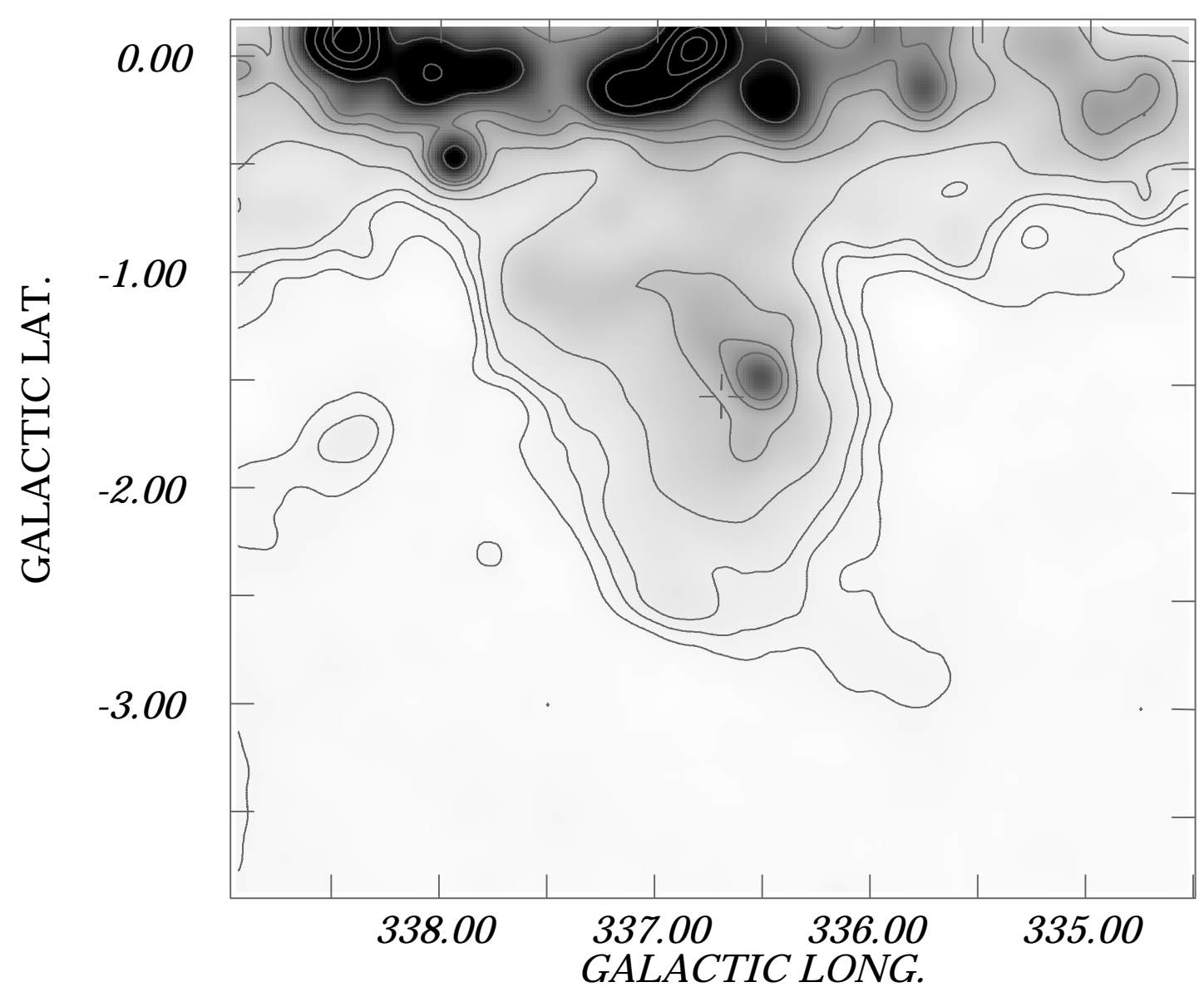

Fig. 6. Image of the $2.417 \mathrm{GHz}$ small scale image (grey scale) (Duncan et al. 1995). The grey scale minimun and maximum are -0.4 and $30 \mathrm{Jy} /$ beam, respectively. The lowest two contour levels are $0.36 \mathrm{~K}(3 \sigma)$ and $0.72 \mathrm{~K}$. The next contour level is $1.08 \mathrm{~K}$ and from there onwards the contour level spacing is $1.08 \mathrm{~K}$. The position of HD $150135 / 6$ is marked by a cross.

patches located further away from the interface, and both depict a radial velocity, $-23.8 \mathrm{~km} \mathrm{~s}^{-1}$, that is similar to that of feature E. The molecular emission seen along the dark cloud delimiting NGC 6188 achieves its maximum intensity at $(l, b)=$ $\left(336^{\circ} .75,-1.25\right)$ (Feature D) and the extreme side closer to the galactic plane is much broader than the one observed in Fig. 8a. This broadening may be due to an increasing contribution from feature B.

In the velocity interval from -23.5 to $-21.9 \mathrm{~km} \mathrm{~s}^{-1}$ (Fig. 8c) the $\mathrm{CO}$ emission extends further away from the galactic plane. A new feature sets in at $(l, b)=\left(336^{\circ} .39,-1^{\circ} .65\right)$. In this velocity range, this new feature shows the distribution in the plane of the sky of the negative velocity wing from the $\mathrm{CO}$ defining feature H. Only a narrow throat of molecular emission joins feature $\mathrm{D}$ with the main body of emission from objects $\mathrm{C}, \mathrm{E}$ and $F$. Though feature $B$ still remains visible, it begins to be distorted by the molecular emission arising from feature $\mathrm{G}$.

In the last velocity range (Fig. 8d) there is almost no CO emission observed along NGC 6188. Only a small contribution from the positive velocity wing of the line arising from feature $\mathrm{B}$ is still observable. The dominant structure is now located some $20^{\prime}$ away from NGC 6193 along a position angle of $\sim 205^{\circ}$. The mean radial velocity of this feature is $-20 \mathrm{~km} \mathrm{~s}^{-1}$ and its southern extension marks the location of feature I.
At more positive velocities (see Fig. 4) though new $\mathrm{CO}$ features are observed, they are located at a projected angular distance of $\sim 45^{\prime}(\sim 17.5 \mathrm{pc}$ at $1300 \mathrm{pc})$ from NGC 6193 and its possible physical link to the Ara OB1 complex becomes increasingly difficult to assess.

It is worth mentioning that a mean $\mathrm{CO}$ spectrum (Fig. 9) of feature $\mathrm{O}$ shows a peak velocity at $-17.5 \mathrm{~km} \mathrm{~s}^{-1}$ and a $F W H M$ of $5.5 \mathrm{~km} \mathrm{~s}^{-1}$. This feature is very similar to the one observed by Phillips et al. (1986) in the ${ }^{12} \mathrm{CO} J=2 \rightarrow 1$ transition. Though in the 1.3-mm transition this spectral feature arises from an isolated $\mathrm{CO}$ cloud, our coarse angular resolution prevent us from establishing whether the 2.6-mm emission arises from a spatially isolated cloud or else originates in a much more extended feature.

Taking into account that the O-stars HD 150135/6 are the main ionizing sources of the difuse HII region RCW 108, that NGC 6188 signals the interface between this HII region and a prominent dust lane seen nearby, and bearing in mind the close agreement in radial velocity among the different line observations, we conclude that all these objects are located at a common distance.

Henceforth, based on the kinematical and morphological evidence presented above, we conclude that the $\mathrm{CO}$ features labelled A, B, C, D, E, F, H, I and O represent the bulk of 


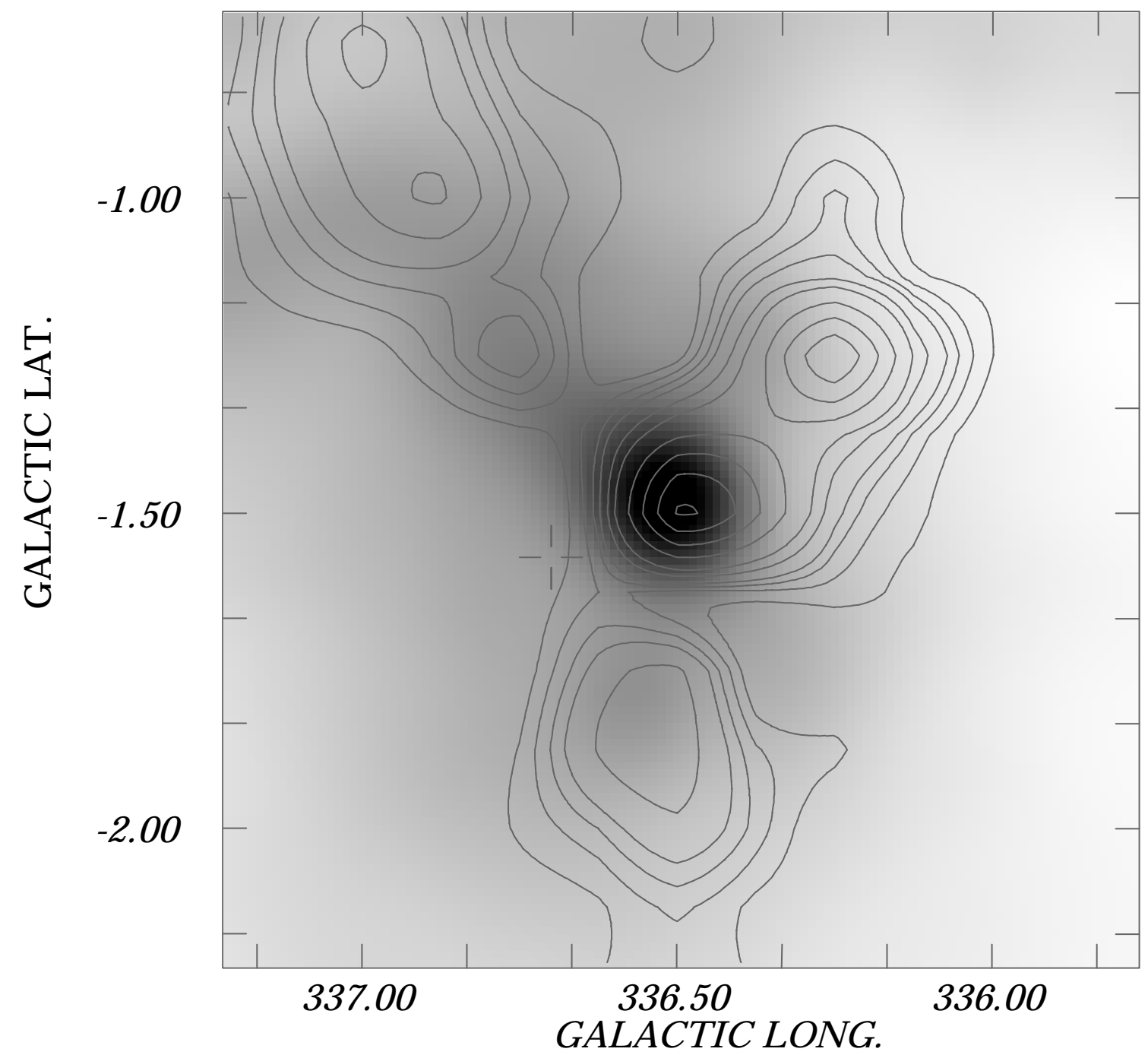

Fig. 7. Overlay of the $2.417 \mathrm{GHz}$ emission (grey scale) and the mean CO emission along the velocity range -25.0 to $-21.0 \mathrm{~km} \mathrm{~s}^{-1}$. The grey scale minimum and maximum are -0.4 and $20 \mathrm{Jy} /$ beam. The lowest countour level is $0.25 \mathrm{~K}(8 \sigma)$ and the next contour levels are $0.5,0.75,1.0$, 1.5 and $2 \mathrm{~K}$. From there onwards the contour spacing is $1 \mathrm{~K}$. The position of HD 150135/6 is marked by a large cross. Notice the extremely good positional coincidence between the $\mathrm{CO}$ feature $\mathrm{E}$ (contour lines) and the high obscuration regions visible in the continuum map.

the molecular gas physically related to the OB association Ara OB1.

$$
.5
$$

There seems to be a clear pattern in radial velocity across the area, in the sense that molecular gas in the northern part of the Ara OB1 complex moves at more negative radial velocity than the gas located in its southern part. This may be a consequence of the complex history of interaction that may have taken place in the region (Arnal et al. 1987; Rizzo \& Bajaja 1994). This particular point will be addressed in a future work to be published elsewhere.

\subsection{Physical and dynamical properties of the molecular gas}

The observed properties and derived physical parameters of the molecular concentrations likely to be genetically associated with Ara OB1 are given in Table 4. A letter identifying the molecular concentration is given in the first column. For com- pletness the corresponding galactic coordinates are taken from Table 1 and are reproduced in the second and third column of Table 4 . The excitation temperature, $T_{\mathrm{ex}}$, (fourth column) is derived from the optically thick ${ }^{12} \mathrm{CO}(J=1 \rightarrow 0)$ emission. The line width, $\Delta V$, given in the fifth column of Table 4 is derived from a Gaussian fitting to the mean $\mathrm{CO}$ emission profile. The latter is obtained by averaging all of the spectra within a molecular cloud. The mean radial velocity of each concentration, $V_{m}$, is given in column sixth. The major $(L)$ and minor $(l)$ axis, seventh and eighth columns, are corrected for beam broadening.

Using the integrated $\mathrm{CO}$ line intensity, $W_{\mathrm{CO}}$, and a $\mathrm{H}_{2}$ column density to $W_{\mathrm{CO}}$ ratio of $(2.3 \pm 0.3) \times$ $10^{20}$ molecules $\mathrm{cm}^{-2}\left(\mathrm{~K} \mathrm{~km} \mathrm{~s}^{-1}\right)^{-1}$ (Strong et al. 1988), the total mass of the molecular clouds can be derived. However, this $\mathrm{H}_{2}$ column density to $W_{\mathrm{CO}}$ ratio has to be scaled down by 0.82 from the published value by Strong et al. 1988, to account for the different calibration scale (Bronfman et al. 1988) of the database from which it was derived, giving a ratio of $1.9 \times 10^{20}$ molecules $\mathrm{cm}^{-2}\left(\mathrm{~K} \mathrm{~km} \mathrm{~s}^{-1}\right)^{-1}$ for the inner Galaxy 

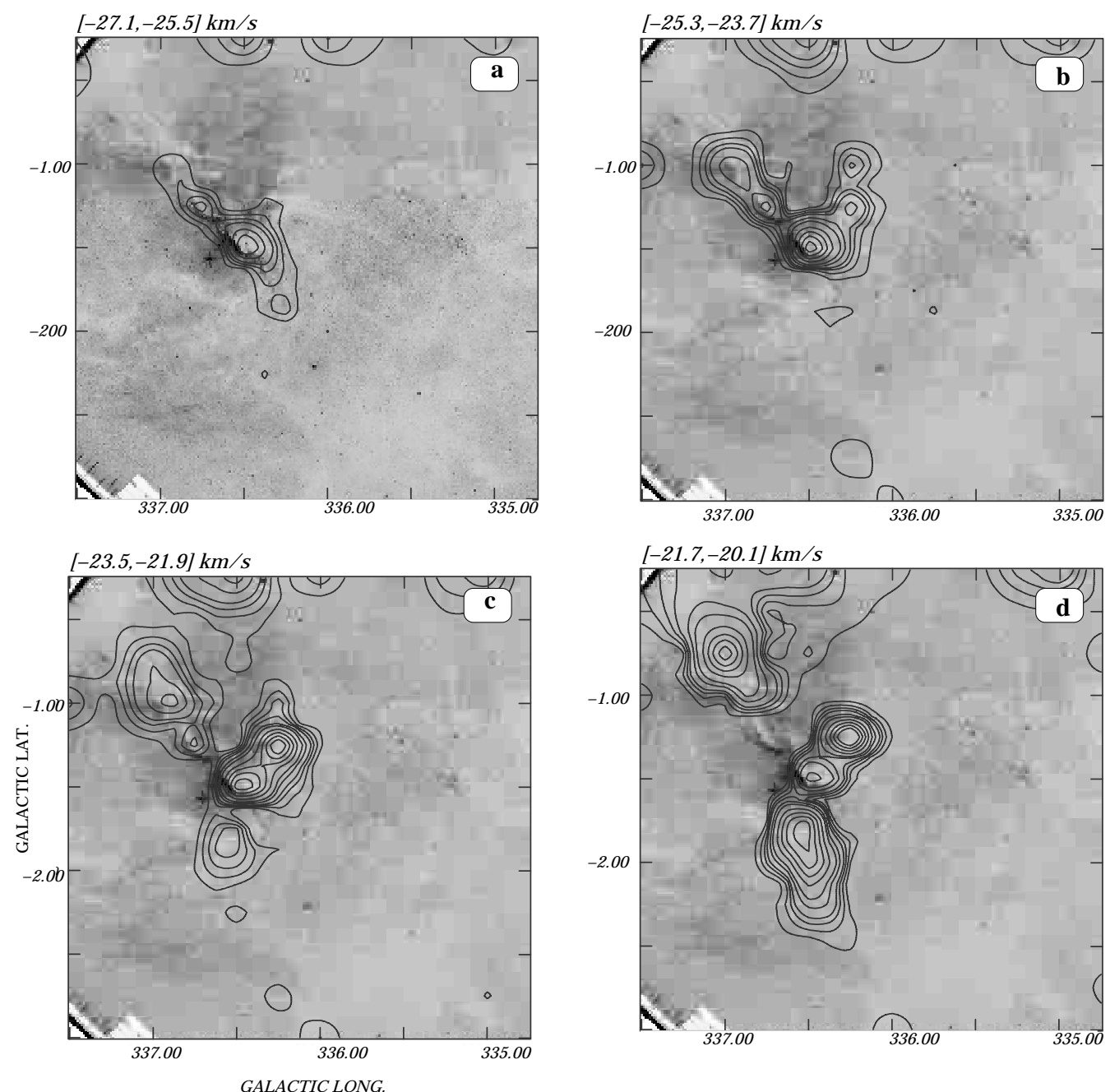

Fig. 8. Overlay of a MAMA R-image (grey scale) and the CO distribution in four selected velocity ranges. The channel width is $1.8 \mathrm{~km} \mathrm{~s}^{-1}$. The central velocity of each image is given in the upper left corner of each figure. The lowest contour level is $0.25 \mathrm{~K}$. The next contours are $0.5,0.75,1.0,1.5$ and $2.0 \mathrm{~K}$. From there onwards the countour spacing is $1 \mathrm{~K}$. The position of HD 150135/6 is marked by a cross. Notice the extremely good positional coincidence between the $\mathrm{CO}$ maxima and the high obscuration regions visible in the infrared image.

(Murphy \& May 1991; Mauersberger et al. 1996). Therefore, adopting a mean molecular weight per $\mathrm{H}_{2}$ of $2.76 m_{\mathrm{H}}$, the total mass given in the ninth column of Table 4 is derived. A mean volume density, tenth column, is derived from the ratio of the total molecular mass and the volume of an ellipsoid whose major and minor axis are $L$ and $l$, respectively.

There is a clear spatial correlation between the $F W H M$ of the $\mathrm{CO}$ line of a given concentration and its angular distance to AraOB1. The broadest profiles are observed towards feature E, the closest concentration to AraOB1, where the FWHM is of the order of $4.6 \mathrm{~km} \mathrm{~s}^{-1}$, while in regions located further away typical $F W H M$ figures fall in the range from 2.4 to $3.0 \mathrm{~km} \mathrm{~s}^{-1}$.

\section{Conclusions}

We have observed the ${ }^{12} \mathrm{CO}(J=1 \rightarrow 0)$ line towards an area of $2.75 \times 3.0$ covering the whole of the OB-association Ara OB1. The main results of this paper are the followings:

a) A total of sixteen molecular clouds were identified across the surveyed area. Based on both morphological and kinematical arguments, nine out of those sixteen concentrations are likely to be associated with the obscuring material observed in the vicinity of NGC 6193.

b) The total molecular mass related to Ara OB1 amounts to $\sim 1.5 \times 10^{4} M_{\odot}$. Individual cloud masses and average cloud densities vary between $180 \leq M \leq 5800 M_{\odot}$ and about $10^{2} \leq n \leq 10^{4}$ molecules $\mathrm{cm}^{-3}$, respectively.

c) The radial velocity of the majority of the $\mathrm{CO}$ structures associated with Ara OB1 falls in the narrow velocity range -24 to $-20 \mathrm{~km} \mathrm{~s}^{-1}$. Concentrations labelled $\mathrm{A}$ and $\mathrm{O}$, seen in projection close to NGC 6193, depict the highest negative $\left(-28.5 \mathrm{~km} \mathrm{~s}^{-1}\right)$ and positive $\left(-17.5 \mathrm{~km} \mathrm{~s}^{-1}\right)$ velocities, respectively. The complex kinematical history of this region (e.g.: Arnal et al. 1987; Rizzo \& Bajaja 1994) may explain our findings.

d) The molecular concentrations E, F and $\mathrm{H}$ have a strong counterpart at 60 and $100 \mu \mathrm{m}$. The dust lane adjacent to the Rim nebula ( $\equiv$ NGC 6188) is also observable at infrared wavelengths.

e) Concentration E coincides with a strong point-like source seen in the radio continuum survey at $2.417 \mathrm{GHz}$ 
Table 4. Parameters of the main CO features associated with Ara OB1.

\begin{tabular}{ccccccccrr}
\hline \hline Feature & $\begin{array}{c}l \\
\left({ }^{\circ}\right)\end{array}$ & $\begin{array}{c}c \\
\left({ }^{\circ}\right)\end{array}$ & $\begin{array}{c}T_{\text {exc }} \\
(\mathrm{K})\end{array}$ & $\begin{array}{c}\Delta V \\
\left(\mathrm{~km} \mathrm{~s}^{-1}\right)\end{array}$ & $\begin{array}{c}V_{m} \\
\left(\mathrm{~km} \mathrm{~s}^{-1}\right)\end{array}$ & $\begin{array}{c}L \\
(\mathrm{pc})\end{array}$ & $\begin{array}{c}l \\
(\mathrm{pc})\end{array}$ & $\begin{array}{r}M \\
\left(10^{3} \times M_{\odot}\right)\end{array}$ & $\begin{array}{r}n \\
\left(10^{3} \times \mathrm{cm}^{-3}\right)\end{array}$ \\
\hline A & 336.38 & -1.88 & 4.0 & 3.2 & -28.5 & 4.3 & 2.7 & 0.18 & 0.22 \\
B & 336.94 & -1.00 & 5.2 & 3.0 & -24.0 & 5.7 & 4.3 & 0.84 & 0.30 \\
C & 336.25 & -1.00 & 5.4 & 1.5 & -23.8 & 1.3 & 2.6 & 0.18 & 1.60 \\
D & 336.75 & -1.25 & 5.5 & 3.4 & -24.6 & 2.7 & 2.3 & 0.41 & 1.10 \\
E & 336.44 & -1.50 & 9.5 & 4.6 & -23.5 & 4.7 & 2.7 & 3.90 & 4.40 \\
F & 336.25 & -1.25 & 13.4 & 3.0 & -21.8 & 4.0 & 2.3 & 2.90 & 5.30 \\
H & 336.56 & -1.88 & 10.3 & 3.2 & -20.0 & 10.7 & 6.2 & 5.80 & 0.56 \\
I & 336.50 & -2.13 & 8.2 & 2.7 & -19.8 & 5.0 & 4.0 & 0.60 & 0.30 \\
O & 336.69 & -1.63 & 5.6 & 5.5 & -17.5 & 7.3 & 4.4 & 0.54 & 0.09 \\
\hline
\end{tabular}

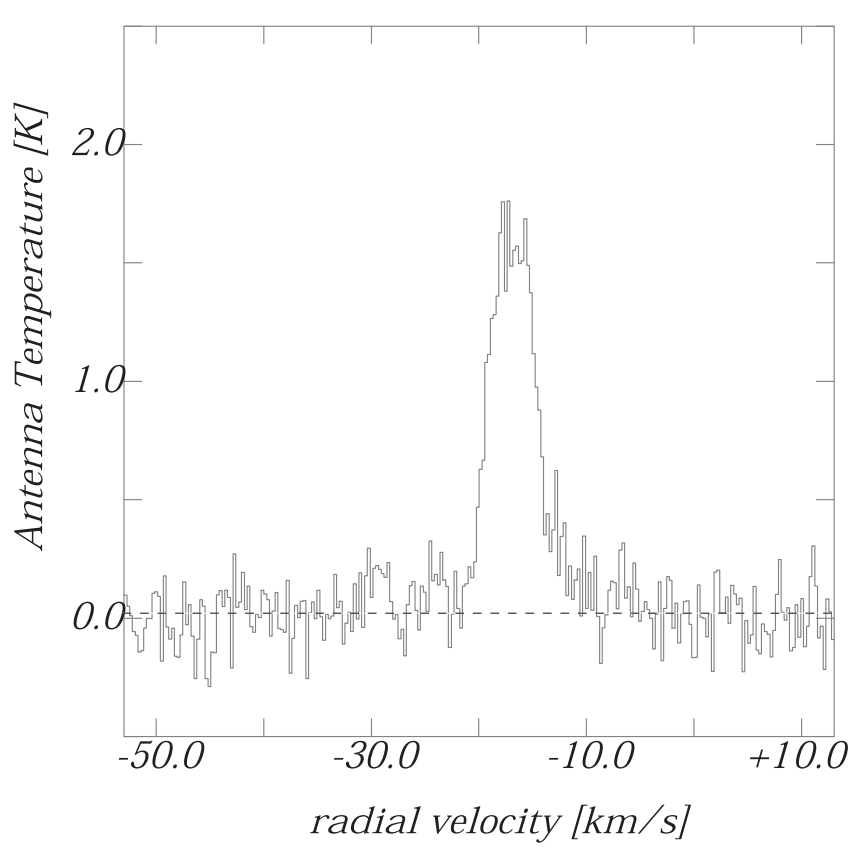

Fig. 9. Mean ${ }^{12} \mathrm{CO}(J=1 \rightarrow 0)$ profile obtained at the position observed by Phillips et al. (1986). The profile units are $T_{A}^{*}$ in kelvin units (ordinate) and $V_{\mathrm{LSR}}$ in $\mathrm{km} \mathrm{s}^{-1}$ (abscissae).

(Duncan et al. 1995). This source was first detected by Goss \& Shaver (1970) at $5 \mathrm{GHz}$ as a high emission measure compact HII region. The peak of this radio continuum source is also coincident with the strong IRAS point source 16362-4845. Very likely the IRAS source and the compact HII region are different observational manifestations of a star forming process that is going on in the interior of molecular concentration $\mathrm{E}$.

f) Extended continuum emission possibly originated in the ionized surface layers of the Ara OB1 molecular complex is observed. Its total flux density at $2.417 \mathrm{GHz}$ is $210 \pm 23 \mathrm{Jy}$, and this large scale thermal continuum emission can be accounted for by the ionization produced by the Lyman continuum photons emitted by the massive stars of NGC 6193.

g) IRAS point sources are also seen projected onto ${ }^{12} \mathrm{CO}$ concentrations $\mathrm{F}$ and $\mathrm{H}$. Likewise in the case of
IRAS 16362-4845, these infrared sources may be indicating that the star-forming process is a widespread phenomenon in this molecular complex.

Acknowledgements. The work of E.M.A. was partially financed by the Consejo Nacional de Investigaciones Científicas y Técnicas (CONICET) of Argentina under Project PIP 4252/96, and by Fundación Antorchas of Argentina through Project 13622/10. The work of Dr. J. R. Rizzo at early stages of this project is gratefully acknowledged. The support of Dr. M. Smith, Director of Cerro Tololo Inter-American Observatory, to the operation of the 1.2-m radiotelescope is highly appreciated. We thank J. Aparici and F. Olmos for assistance with the observations and maintenance of the radiotelescope. It is a pleasure to thank Dr. C. Cappa for her valuable comments on an early version of this paper. J.M. acknowledges the support from FONDECYT (Chile) grant 1010431 and from the Chilean Centro de Astrofísica FONDAP 15010003.

\section{References}

Arnal, E. M., Cersósimo, J. C., May, J., \& Bronfman, L. 1987, A\&A, 174,78

Arnal, E. M., Morrel, N. I., García, B., \& Levato, O. H. 1988, PASP, 100, 1076

Brand, J., \& Blitz, L. 1993, A\&A, 275, 67

Bronfman, L., Cohen, R. S., Alvarez, H., May, J., \& Thaddeus, P. 1988, ApJ, 324, 248

Bronfman, L., Alvarez, H., Cohen, R. S., \& Thaddeus, P. 1989, ApJ, 71,481

Caswell, J. L., \& Robinson, B. J. 1974, Austr. J. Phys., 27, 597

Caswell, J. L., \& Haynes, R. F. 1987, A\&A, 171, 261

Cersósimo, J. C. 1982, ApJ, 22, L157

Chaisson, E. J. 1976, in Frontiers of Astrophysics, ed. E. H. Avrett (Cambridge: Harvard Univ. Press), 259

Duncan, A. R., Stewart, R. T., Haynes, R. F., \& Jones, K. L. 1995, MNRAS, 277, 36

Fitzgerald, M. P. 1987, MNRAS, 229, 227

Frogel, J. A., \& Persson, S. E. 1974, ApJ, 214, 725

Gillespie, A. R., Huggins, P. J., Sollner, T. C. L. G., et al. 1977, A\&A, 60, 221

Georgelin, Y. P., \& Georgelin, Y. M. 1970, A\&A, 6, 349

Georgelin, Y. M., Russeil, D., Marcelin, M., et al. 1996, A\&AS, 120, 41

Goss, W. M., \& Shaver, P. A. 1970, Austr. J. Phys., Ap. Suppl. 14, 1 
Fich, M., \& Terebey, S. 1996, ApJ, 472, 624

Herbst, W. 1975, AJ, 80, 212

Herbst, W., \& Havlen, R. J. 1977, A\&AS, 30, 279

Henning, T. 1996, in The Cosmic Dust Connection, ed. J. M. Greenberg (Dordrecht: Kluwer Academic Publishers)

Kaltcheva, N. T., \& Georgiev, L. N. 1992, MNRAS, 259, 166

Kutner, M. L., \& Ulich, B. L. 1981, ApJ, 250, 341

Mauersberger, R., Henkel, C., Wielebinski, R., Wiklind, T., \& Reuter, H.-P. 1996, A\&A, 305, 421

Melnick, J. 1992, in Star Formation in Stellar Systems, III Canary

Islands Winter School of Astrophysics, ed. G. Tenorio-Tagle,

M. Prieto, \& F. Sánchez (Cambridge: University Press)

Moffat, A. F. J., \& Vogt, N. 1973, A\&AS, 10, 135

Murphy, D. C., \& May, J. 1991, A\&A, 247, 202

Phillips, J. P., de Vries, C. P., \& de Graauw, T. 1986, A\&AS, 65, 465

Reich, P., Testori, J. C., \& Reich, W. 2001, A\&A, 376, 861

Rizzo, J. R., \& Bajaja, E. 1994, A\&A, 332, 1025
Rodgers, A. W., Campbell, C. T., \& Whiteoak, J. B. 1960, MNRAS, 121,103

Schaerer, D., \& de Koter, A. 1997, A\&A, 322, 598

Shaver, P. A., \& Goss, W. M. 1970, Austr. J. Phys., Ap. Suppl. 14, 77

Straw, S., Hyland, A. R., Jones, T. J., et al. 1987, ApJ, 314, 283

Strong, A. W., Bloemen, J. B. G. M., Dame, T. M., et al. 1988, A\&A, 207, 1

Yamaguchi, R., Saito, H., Mizuno, N., et al. 1999, PASJ, 51, 791

Waldhausen, S., Martínez, R., \& Feinstein, C. 2000, AJ, 117, 2882

Walker, H. J., Cohen, M., Volk, K., Wainscoat, R. J., \& Schwartz, D. E. 1989, AJ, 98, 2163

Whiteoak, J. B. 1963, MNRAS, 125, 105

Whiteoak, J. B., \& Gardner, F. F. 1974, A\&A, 37, 389

Whiteoak, J. B., Otrupcek, R. E., \& Rennie, C. J. 1982, Proc. ASA, 4, 434

Wilson, T. L., Mezger, P. G., Gardner, F. F., \& Milne, D. K. 1970, A\&A, 6, 364 